\title{
Fast solute diffusivity in ionic liquids with silyl or siloxane groups studied by the transient grating method
}

Takatsugu Endo, ${ }^{\dagger}$ Shinya Nemugaki, ${ }^{\dagger}$ Yuki Matsushita, ${ }^{\dagger}$ Yasuhiro Sakai, ${ }^{\dagger}$ Hiroaki Ozaki, ${ }^{\dagger}$ Yusuke Hiejima, ${ }^{\dagger}$ Yoshifumi Kimura, ${ }^{\ddagger}$ and Kenji Takahashi ${ }^{\dagger} * *$

'Institute of Science and Engineering, Kanazawa University, Kakuma-machi, Kanazawa, 920-1192, Japan

${ }^{\ddagger}$ Department of Molecular Chemistry and Biochemistry, Faculty of Science and Engineering, Doshisha University, Kyotanabe 610-0321, Japan 


\begin{abstract}
To achieve ionic liquids (ILs) that show fast solute diffusivity independent of viscosity domination, sixteen ILs containing Si or Si-O-Si groups (SilLs) were synthesized. Diffusion coefficients of three solute molecules with different molecular sizes, i.e., $\mathrm{CO}$, diphenylacetylene, and diphenylcyclopropenone, were determined in Sills using the transient grating method and the results were compared to other solvent system. Sills showed distinguishably faster diffusivity for the smallest solute, $\mathrm{CO}$, than conventional ILs at the same viscosity, particularly in the high viscosity region. Based on previous results and our estimation, three plausible factors exists that contribute to the faster solute diffusivity in SilLs, i.e., the flexibility of the $\mathrm{Si}$ or $\mathrm{Si}-\mathrm{O}-\mathrm{Si}$ group, decreased interaction between the cation and the solute, and increased free volume because of the bulky structure.
\end{abstract}




\section{Introduction}

Ionic liquids (ILs) are salts that are liquid at ambient temperature. Since they are composed solely of ions and have several outstanding characteristics as solvents, such as negligible flammability and vapor pressure, high thermal/chemical/electrochemical stabilities, and unique solubility, ILs are potentially useful as new types of chemical reaction media and electrolytes. Considering these applications, solute diffusivity in solvents is a very important factor, as exemplified by diffusion-controlled reactions. Classically, a solute diffusion is represented by the Stokes-Einstein (SE) equation.

$$
D=\frac{k_{B} T}{C \pi \eta r},(1)
$$

where $k_{\mathrm{B}}$ is the Boltzmann constant, $T$ is the temperature, $C$ is a constant theoretically ranging from 4 (slip boundary condition) to 6 (stick boundary condition), $\eta$ is the viscosity, and $r$ is the hydrodynamic radius of the solute.

Since most ILs exhibit high viscosity (typically $10^{1}-10^{3} \mathrm{mPa}$ ) compared to conventional organic solvents (typically $10^{-1}-10^{1} \mathrm{mPa}$ ), designing low-viscosity ILs that facilitate solute movement is a significant challenge that many studies have been devoted. ${ }^{1-5}$ Molecular designs that control ion size, flexibility, and asymmetry of both cations and anions strongly affect the viscosity of ILs. However, attempts to lower the viscosity are limited due to the intrinsically strong Coulombic interactions that make them viscous. Since the SE equation is known to break down particularly for small solute molecules, which display higher diffusivity, we propose another approach to achieve high solute diffusivity in ILs, which is to design ILs that enhance solute diffusivity that are less independent of viscosity than theoretically predicted.

In this sense, we focused on silyl or siloxane structures seen in silicone oil. It is widely known that solute molecules in silicone oil have higher translational and rotational diffusivity than those predicted by the SE equation. ${ }^{6-14}$ For example, the diffusion coefficients of phthalic anhydride in polydimethylsiloxane were estimated from fluorescence quenching rates of the solute, and the values were up to $10^{6}$ times higher than those calculated from the SE equation in the high viscosity region $\left(10^{6} \mathrm{mPa} \mathrm{s}\right){ }^{7}$ High flexibility and large free volume are considered to account for these features of silicone oil. In fact, the introduction of silyl or siloxane structures in ILs (SiILs) was first reported by Shirota et al. ${ }^{15-16}$ Since then, many groups have synthesized new types of SilLs and revealed their characteristics. ${ }^{17-28}$ In the first paper, ${ }^{15}$ a novel imidazolium-based cation with a trimethylsilylmethyl group was synthesized and its physical properties were compared to an IL with a neopentyl group on the cation. The only difference in structure between these ILs is one atom on the side chain, with silane in the trimethylsilylmethyl group and carbon in the neopentyl group. A significant decrease in the viscosity was observed on the substitution of silane. Later, an IL with a siloxane group was synthesized by the same group that showed similar viscosity to the IL with a silyl group. ${ }^{16}$ It was revealed by electron density calculations and Raman-induced Kerr effect spectroscopy that 
the viscosity decrease stemmed from the decrease in cation-anion interactions. Niedermeyer et al. investigated the flexibility of siloxane groups attached to imidazolium cations through density functional theory (DFT) calculations. ${ }^{29}$ Their results showed that the rotational barriers of the side chain were lower for the siloxane group than that for the butyl group. These findings for both silicone oil and $\mathrm{Si}$ or $\mathrm{Si}-\mathrm{O}-\mathrm{Si}$ group containing ILs imply that SilLs are promising in terms of high solute diffusivity.

In this paper, we synthesized sixteen Sills (Figure 1) from four cations, 1-methyl-3propyltrimethylsilylimidazolium (propylSilm), 1-methyl-3-methylpentamethyldisiloxyimidazolium (SiOSilm), N-methyl-N-propyltrimethylsilylpyrrolidinium (propylSiPyrr), and N-methyl-Nmethylpentamethyldisiloxypyrrolidinium (SiOSiPyrr), paired with four anions, bis(fluorosulfonyl)imide (FSI), bis(trifluoromethanesulfonyl)imide (TFSI), bis(pentafluoroethanesulfonyl)imide (BETI), and hexafluorophosphate $\left(\mathrm{PF}_{6}\right)$. The solute diffusions of these SilLs were then compared with those of silicone oils as well as of IL and molecular solvent systems previously reported. ${ }^{30-31}$ Solute diffusions were measured using the transient grating (TG) method. The TG method has been employed to determine the diffusion coefficients of photoreacted and photoproduced molecules in solutions and proven to be effective even in ILs as high-viscosity media. ${ }^{30-32}$ We used diphenylcyclopropenone (DPCP), which produces diphenylacetylene (DPA) and CO by photoreaction (Scheme 1). This means that the diffusion coefficients of three solute molecules with different molecular sizes can be detected simultaneously. The solute diffusivity and factors that dominate the diffusivity in SilLs are discussed based on the (fractional) SE relationship.

\section{Experimental section}

\section{1. Sample preparations}

Sills were synthesized according to a conventional procedure. ${ }^{15}$ All starting materials, i.e., 1methylimidazole (ACROS), $N$-methylpyrrolidine (ACROS), 3-chloropropyltrimethylsilane (Gelest), chloromethylpentamethyldisiloxane (Gelest), potassium bis(fluorosulfonyl)imide (Kanto Kagaku), lithium bis(trifluoromethanesulfonyl)amide (Kanto Kagaku), lithium bis(pentafluoroethanesulfonyl)imide (Kishida Kagaku), and potassium hexafluorophosphate (Kanto Kagaku) were used as received. First, chloride salts were obtained by the quaternization of amine derivatives with $\mathrm{Si}$ or $\mathrm{Si}-\mathrm{O}-\mathrm{Si}$ containing compounds terminated with a chlorine atom. The reactions were typically carried out in acetonitrile at $353 \mathrm{~K}$ for $48 \mathrm{~h}$ under an Ar atmosphere. Appropriate purifications were performed for the obtained chloride salts such as washing with ethyl acetate, recrystallization, and decolorizing with activated charcoal. Sills were prepared from the corresponding chloride salts by ion exchange with a lithium or potassium salt in an appropriate solvent. The obtained liquids were washed with distilled water several times until they passed the $\mathrm{AgNO}_{3}$ 
test and decolorized with activated charcoal to minimize absorbance at $355 \mathrm{~nm}$ (typically less than 0.3 with a path length of $1 \mathrm{~cm})$. A detailed procedure of the preparation of [propylSiIm][FSI] is described in Supporting Information as an example. All SilLs except [propylSiPyrr][FSI] (m.p. =328-333 K), [propylSiPyrr][PF6] (m.p. $=388-393 \mathrm{~K}),[\operatorname{SiOSiPyrro}][\mathrm{BETI}](\mathrm{m} . \mathrm{p} .=333-338 \mathrm{~K})$, and [SiOSiPyrro][PF 6$](\mathrm{m} . \mathrm{p} .=378-383 \mathrm{~K})$ were liquid at room temperature. The final yields ranged from $22 \%$ to $72 \%$. The Sills were characterized by ${ }^{1} \mathrm{H},{ }^{13} \mathrm{C}$, and ${ }^{19} \mathrm{~F}$ NMR spectroscopies (JEOL JNM-ECS400 or JNM-ECA600, see Supporting Information for details). The cation and anion structures of the SilLs are shown in Figure 1. Conventional ILs with alkyl groups (AlILs), 1-allyl-3-ethylimidazolium (AEIm), $N$-methyl- $N$-propylpyrrolidinium (P1,3), and $N$-methyl$N$-butylpyrrolidinium (P1,4), paired with TFSI anions were purchased from Kanto Kagaku. Silicone oils as reference materials with various viscosities (i.e., KF-96-10CS, KF-96-50CS, KF-96-100CS, and KF-96500CS, the numbers expressed as xxCS are kinematic viscosity at $298 \mathrm{~K}$ ) were purchased from Shin-Etsu Silicone. The samples were dried under vacuum overnight before use.

\section{2. Viscosity and Density measurements}

Viscosity of the ILs were measured with a cone/plate viscometer (Brookfield LVDV-II). For room temperature measurements, the temperature was stabilized at $296 \pm 1 \mathrm{~K}$. The temperature was controlled between $294.2 \mathrm{~K}$ and $333.2 \mathrm{~K}$ for variable temperature experiments with $0.1 \mathrm{~K}$ accuracy. Density measurements were conducted with Anton Paar, DMA 35 at $298.2 \pm 0.1 \mathrm{~K}$.

\subsection{TG measurements}

For the TG measurements, DPCP was dissolved in the ILs to achieve an absorbance of 1.5 with a 0.5 $\mathrm{cm}$ or $1 \mathrm{~cm}$ optical length cell at $355 \mathrm{~nm}$, which roughly gives a concentration of $10^{-3} \mathrm{M}$. This concentration would be dilute enough to approximate mutual-diffusion coefficients obtained from TG experiments by selfdiffusion coefficients of solute molecules. The sample solution was filtered to remove any undissolved solutes and dust before use.

The experimental setup for TG measurements was similar to those reported elsewhere. ${ }^{31}$ Briefly, the third harmonic pulse ( $355 \mathrm{~nm}$ ) of the neodymium-doped yttrium aluminum garnet (Nd:YAG) laser (LOTIS LS-2144DG) was used as an excitation pulse. This excitation pulse was attenuated with a combination of a $1 / 2 \lambda$ plate and a polarizer, divided into two beams with a beam splitter, and focused on a sample solution at a certain angle $(\theta)$ to produce transient grating in the sample. A $633 \mathrm{~nm}$ He-Ne laser beam (Nippon component LGK-7647J) was used as a probe beam. When the angle of the probe beam satisfies the Bragg condition, the diffracted probe beam is detected by a photomultiplier tube (Hamamatsu H6780-20MOD) as a TG signal. A UV cutoff filter was placed in front of the photomultiplier tube to remove contributions from the excitation light. The signal decays with vanishing interference fringes, which is mainly due to 
photoreacted solute diffusion driven by concentration gradients. These decay data are then sent to a digital oscilloscope (Tektronix TDS 2024B). TG measurements were performed at room temperature (295 $\pm 1 \mathrm{~K})$. For high temperature experiments, the temperature was controlled with a heater surrounding a sample holder within $1 \mathrm{~K}$ accuracy.

\section{4. DFT calculations}

DFT calculations for the ions were performed using the Gaussian 03 program package. ${ }^{33}$ Full geometry optimization analyses in the gas phase were carried out using 6-311+G(d,p) basis sets based on Becke's three-parameter hybrid method ${ }^{34}$ with LYP correlation (B3LYP). ${ }^{35-36}$ No imaginary frequencies were produced by the optimized structures, ensuring the presence of an energetic minimum. Ionic volumes of the ions were estimated using DFT calculations. ${ }^{37}$ The estimated values of the volume based on this procedure can be considered as the van der Waals volume. ${ }^{38}$ Since ionic volume calculations in the program are based on the Monte Carlo method, such calculations were repeated 15 times and the average values were reported. The ionic structures used for volume calculations are shown in Figure S1.

\section{Results and Discussion}

\section{1. Viscosity and Density of ILs}

The measured viscosities and densities of the ILs as well as the silicone oils are summarized in Table 1. Although caution needs to be exercised when comparing viscosities of ILs because of the diversity of the ion structures, generally SilLs have higher viscosities than AlILs. This is because the bulky structure of the silyl or siloxane group slows down the movement of the cation even though the interaction between the cation and anion and the flexibility of the side chain can have an opposite effect on the viscosity. ${ }^{15-16,29}$ This idea is supported by the SilL diffusion coefficients measured by Chung et al. ${ }^{39}$ They found that the diffusivity of the trimethylsilylmethyl-substituted imidazolium cation is nearly identical to that of the TFSI anion, whereas cations of ILs generally move faster in AlILs. ${ }^{4043} \mathrm{ILs}$ with the Si-O-Si group showed somewhat lower viscosities than those with the Si group, as was previously reported. ${ }^{16}$ Except $\mathrm{PF}_{6}$ anion, pairing with a smaller anion or aromatic cation causes lower viscosity, which is again in line with previous AlIL results. ${ }^{445}$ The density values in Table 1 can be utilized to derive free volume fractions of the ILs (vide infra).

\section{2. TG signal}

Figure 2 (a) shows the TG signal of solute diffusion in [propylSiIm][FSI]. The TG signals is quite similar to those observed in other ILs. ${ }^{30-31}$ After the decay of the thermal grating signal in microsecond time 
region, three slower components due to the molecular species could be identified. The TG signals of translational molecular diffusion were simulated by the sum of three exponentials corresponding to three different diffusive species as ${ }^{30-31}$

$$
\begin{aligned}
& I_{T G}(t) \propto\left\{\Delta n_{C O} \exp \left(-t / \tau_{C O}\right)+\Delta n_{D P A} \exp \left(-t / \tau_{D P A}\right)+\Delta n_{D P C P} \exp \left(-t / \tau_{D P C P}\right)\right\}^{2} \\
& 1 / \tau_{i}=D_{i} q^{2}
\end{aligned}
$$

with the assumption that $\tau_{\mathrm{CO}} \ll \tau_{\mathrm{DPA}}<\tau_{\mathrm{DPCP}}$, following the order of molecular size. $\Delta n_{\mathrm{CO}}, \Delta n_{\mathrm{DPA}}$, and $\Delta n_{\mathrm{DPCP}}$ are the initial peak-null differences of the refractive indices because of species grating. $D_{\mathrm{CO}}, D_{\mathrm{DPA}}$, and $D_{\mathrm{DPCP}}$ are the translational diffusion coefficients. For fitting, we fixed the ratio of $\Delta n_{\mathrm{DPCP}}: \Delta n_{\mathrm{DPA}}$ as 1.15: -1 , as was done in a previous paper. ${ }^{30}$ The solid line in Figure 2 (a) shows the fitting result, which demonstrates that eq. (2) was well-suited to simulate the time profile. The diffusion coefficients were determined by the slope of the plot of $\tau^{-1}$ against $q^{2}$ where $q$ is the grating lattice wavenumber defined by

$$
q=\frac{4 \pi \sin (\theta / 2)}{\lambda_{e x}}
$$

Here $\theta$ and $\lambda_{\text {ex }}$ are the crossing angle and the wavelength of the excitation pulse, respectively. The value of $q$ was determined by the decay rate of the thermal grating signal $1 / \tau_{\mathrm{th}}$ using the relation

$$
1 / \tau_{t h}=D_{t h} q^{2}
$$

where $D_{\text {th }}$ is the thermal diffusivity of solvent. The thermal grating signal was obtained by measuring the TG signal of bromocresol purple in ethanol measured at the same optical geometry and using the literature value of $D_{\mathrm{th}}$ for ethanol calculated by $D_{\mathrm{th}}=\lambda \rho C_{\mathrm{p}}$; here $\lambda$ is the thermal conductivity, $\rho$ is the density and $C_{\mathrm{p}}$ is the isobaric heat capacity. ${ }^{30}$

Typical plots are shown in Figure 2 (b) and (c) for [propylSilm][FSI]. The plots show that $\tau^{-1}$ is linearly dependent on $q^{2}$, intercepting at 0 . The diffusion coefficients determined in this manner are summarized in Tables S1 and S3.

\section{3. Diffusion coefficients and comparison to previous results}

Since the ILs used have different chemical structures and viscosities, the diffusion coefficient values should not be compared directly. Therefore, the SE plots, i.e., the sample diffusion coefficients versus $T / \eta$, were used. Figure 3 includes the diffusion coefficients obtained in this study at $296 \mathrm{~K}$ and the data are compared to the previous results ${ }^{30-31}$ taken at room temperature. It can be seen that solute diffusions in silicone oils deviated considerably and were faster than those predicted from the SE equation. The diffusion coefficients for $\mathrm{CO}$ are up to 700 times faster in the most viscous silicone oil. The deviation and fast 
diffusivity of solute diffusions are more prominent with smaller solutes. This deviation can be parametrized with $p$ in the following fractional SE equation.

$$
D=D_{0}\left(\frac{T}{\eta}\right)^{p}
$$

When $p$ is equal to 1, eq. (6) corresponds to the SE equation. There are many factors that contribute to lowering parameter $p$, such as the ratio of solute-solvent size and their interactions. ${ }^{30-31,46-49}$ Also, structural and/or dynamic heterogeneity have been discussed for a wide range of neat liquids. ${ }^{50-54}$ In the silicone oils, $p$ values were estimated to be $0.09,0.14$, and 0.22 for CO, DPA, and DPCP, respectively (see Table S2), with the smaller solutes showing larger deviation from the SE equation. Even though the trend of $p$ values is consistent with previous results for AlILs and their mixtures with molecular solvents shown in Figure 3, there is a significant gap in the absolute values (i.e., $0.66,0.89$, and 0.92 for CO, DPA, and DPCP in the AIIL system, respectively). A smaller $p$ indicates that it is less temperature- and viscosity-dependent and maintains high solute diffusivity even in the viscous state. The results for silicone oils with extremely low $p$ show promise for successful introduction of the siloxane group into ILs that generally tend to have high viscosity. High solute translational and rotational diffusivities in silicone oil have been previously reported. ${ }^{6-14}$ The authors attributed these characteristics to the high flexibility of the chain and the large free volume due to siloxane groups.

Although the plots of all types of the ILs seem to deviate from the SE equation, the degree is much less than for silicone oils. In particular, the diffusion coefficients of the largest solute molecule, DPCP, may seem to lie in the SE equation assuming the slip boundary $(C=4)$ in the ILs. The green symbols in the figure represent the solute diffusion coefficients in ILs constituting phosphonium cation with long alkly chains (PhILs). They show faster solute diffusions than the AlILs and even the SILs. The fast diffusion in the PhILs was already reported ${ }^{31,55}$ and connected with high flexibility of the chains, large cation size and also structural heterogeneity. Structural heterogeneity in ILs, i.e., forming nanostructure with polar (charged parts) and non-polar (alkyl chains) domains, is an important issue in researches on ILs, even though it is beyond the scope of this paper. The fitting curves of the PhILs are above those of the Sills and AlILs in the T/ $\eta$ range investigated here.

Comparing the results from the SilLs with those from the AlILs, the fitted curve for the former is departed from the one for the latter, and shows faster diffusivity in the smaller $T / \eta$ region. This trend is more significant in the smallest solute CO. Although it is still the case that the diffusion in the SilLs is slower than in the $\mathrm{PhILs}$ in the temperature range investigated, the degree of the deviation from the SE equation, expressed by the $p$ value, is smaller in the former, which indicates that the trend would be reversed in more viscous region. Judging from the slopes ( $p$ value) for the CO diffusion, one may find that the SilLs $(p=0.22)$ 
have a similarity to the silicone oils ( $p=0.09)$, rather than the PhIL $(p=0.43)$ and AlIL $(p=0.66)$ systems. This would demonstrate the successful inheritance of the characteristic solute-diffusion property from the silicone oils to the Sills.

The SE equation assumes large particle diffusion compared to the molecular size of the medium. Solute molecules comparable or even smaller in size than the solvent, as in our case, require a more sophisticated approach to discuss the "deviation," as the size ratio is known to strongly contribute to $p . .^{30-31,49,51}$ Recently, the volume ratio of solute to solvent molecules was demonstrated to be one important factor for solute diffusion in ILs. ${ }^{31,48}$ In addition, bulky silyl or siloxane groups result in larger molecular radii of the SilLs (vide infra). In such cases, the Gierer-Wirtz (GW) equation can be introduced ${ }^{56}$

$$
\begin{aligned}
& D=\frac{k_{B} T}{C \pi \eta r \xi} \\
& \xi=\left(2 \frac{r_{m}}{r}+\frac{1}{1+r_{m} / r}\right)^{-1}
\end{aligned}
$$

where $r_{\mathrm{m}}$ is the molecular radius of the solvent. Here, the average radii of cations and anions were used for $r_{\mathrm{m}}$. The diffusion coefficients taking into account the solute-solvent size ratio $(D \zeta)$ are depicted in Figure S2. The introduction of the solute-solvent size ratio into the SE equation hardly change the gap ( $p=0.21$ and 0.62 for Sills and AlILs, respectively), demonstrating that the higher solute diffusivity of the SiILs in the low $T / \eta$ region would not come from the large cation size of the Sills with the bulky side chain.

In order to see how the dependence on $T / \eta$ changes using the same IL, we also measured temperature effect on diffusion as shown in Figure 4. To highlight the side chain effect, we selected two SilLs, [propylSiIm][TFSI] and [SiOSiIm][TFSI], and three AlILs, [BMIm][TFSI], [EMIm][TFSI] and [AEIm][TFSI], with the same cation ring and anion structures. We also studied the temperature dependence in silicone oils and PhILs, where the faster diffusion is detected. For [BMIm][TFSI], [EMIm][TFSI], and $\mathrm{PhILs}$, the data are taken from $\mathrm{ref}^{31}$. All solvent groups including the silicone oils provide similar $p$ values, represented as slope in the figure, in each solute case, differing from the results at room temperature. The $p$ values of the solvents are significantly larger than the ones from the experiments at room temperature and become comparable each other. This more SE equation-following behavior is simply attributed to the change of solute diffusivity itself by temperature. Temperature increase does not only change viscosity but also directly enhance solute diffusivity, unlike change of ion species (Figure 3). It should be noted that there is still a difference in $p$ value among the solvents (Table S2). It is still the case that the deviation from the SE equation is larger in the Sills than the AlILs. On the other hand, $p$ in the PhILs and silicone oils obtained from various temperature experiments shows a different trend from the one obtained from Figure 3. The solute-solvent size ratio was again incorporated into the SE equation and the results are given in Figure S3. 
As in Figure S2 and Table S2, the GW theory does not change the difference in $p$ (Table S2). Even though the GW theory would not completely remove the solute size effect, no influence on the difference in $p$ strongly suggests that the solute diffusivity in each class of the ILs is governed by the different factors. Since some solute diffusion mechanisms would be highly dependent on temperature and the others are not so much, the difference can be a clue to unravel diffusion mechanism for each solvent. However, a reasonable speculation would not be achieved at this stage because of the lack of accurate assignments of diffusion mechanism for all the solvent groups.

\section{4. Plausible factors contributing small $p$ for $\mathrm{CO}$ diffusion in Sills}

The solute diffusivities in Sills are not comparable to those in silicone oil. However, particularly for $\mathrm{CO}$, the diffusivity in SilLs is slightly larger than in AlILs. This is the case of the results with a variety of ILs and at various temperatures with ILs constituting the same anion and the cation ring structure. This indicates the presence of the difference in factors that control the small solute diffusion between Sills and AlILs. Since utilizing the GW theory, which takes into account the solute-solvent size ratio, does not eliminate the difference in diffusion, it is reasonable to attribute this faster diffusion to other molecular-level properties.

As we first expected, there are three possible factors, that is, flexibility, interaction energy and free volume. The high flexibility and large free volume originate from the siloxane (or silyl) structure. ${ }^{6-9,14-15}$ The change in intermolecular interaction is caused by change in ionic nature of the SiIL ions. ${ }^{15-16,29}$ Hereafter, this discussion is framed in terms of these three factors. As previously suggested by Shirota et al., the flexibility of the silyl and siloxane groups affect the physical properties of ILs. ${ }^{15-16}$ Later, Niedermeyer et al. confirmed the high flexibility of the SiOSiIm cation compared to the BMIm cation through DFT calculations. ${ }^{29}$ They obtained potential energy surfaces of the rotations along each bond angle in the side chain and found lower activation energies than the butyl group rotation for the same cation ring structure. They also discovered rotational coupling within the $\mathrm{Si}-\mathrm{O}-\mathrm{Si}$ chain, which facilitated the movement of surrounding ions and molecules. The flexible side chain can decrease the local viscosity of solutes and consequently enhance solute diffusivities, as explained by the SE equation.

Although there have been no reports regarding the interaction between neutral solutes and ions of SilLs, electron density calculations and Raman-induced Kerr effect spectroscopy revealed a decrease in cationanion interactions. ${ }^{15-16}$ Niedermeyer et al. estimated the cation-anion interactions of the SiOSiIm and BMIm cations with the chloride anion in the gas phase. ${ }^{29}$ They concluded that the interaction energies are nearly identical; however, a slight decrease was reported as $367.23 \mathrm{~kJ} \mathrm{~mol}^{-1}$ for SiOSiIm/Cl and $377.99 \mathrm{~kJ} \mathrm{~mol}^{-1}$

for $\mathrm{BMIm} / \mathrm{Cl}$. The typical ion-ion interaction potential is expressed as $U_{\text {ion-ion }}=-q_{+} q_{-} / 4 \pi \varepsilon_{0} \varepsilon_{r} r_{d}^{2}$, where $q_{ \pm}$is the charge, $\varepsilon_{0}$ and $\varepsilon_{\mathrm{r}}$ are the dielectric constants in the gas phase and medium, respectively, and $r_{\mathrm{d}}$ is the 
distance between two ions. The interaction of an ion and either a permanent or induced dipole has a very similar form, where dipole moment $(\mu)$ is used instead of charge. Based on this primitive approach, perhaps slightly weaker solute - solvent interactions in SilLs compared to AlILs is responsible for their different values of $p$.

Regardless of the silyl or siloxane containing side chain, free volume in solvents is an important contributor to the diffusivity, as classically presented in the Cohen-Tumbull equation. ${ }^{57}$ However, unlike the other two factors, free volume has not been investigated in Sills. Here, we derived the free volume fraction defined as

$$
f_{f v}=\frac{V_{F U}-V_{i o n s}}{V_{F U}}
$$

where $V_{\mathrm{FU}}$ is the formula unit volume obtained from molecular weight divided by density and $V_{\text {ions }}$ is the sum of van der Waals volumes of the cation and anion. These results are summarized in Table 2. The free volume fractions of SilLs are generally somewhat larger than in AlILs, which can also explain the faster solute diffusion in SilLs. It should be noted that other trends can also be seen in Table 2. SiILs with a siloxane group may display larger $f_{\mathrm{fv}}$ than that with silane groups. This implies that high $f_{\mathrm{fv}}$ ILs are obtained if a longer $\mathrm{Si}-\mathrm{O}-\mathrm{Si}$ chain is introduced on the $\mathrm{IL}$ cation. This tendency can also be seen in the difference of the anion, where larger (flexible) anions seem to possess larger $f_{\mathrm{fv}}$ values, while it results in undesirably higher viscosity (Table 1).

\section{Conclusion}

We synthesized sixteen ILs containing Si or Si-O-Si groups, in which the diffusion coefficients of CO, DPA, and DPCP were measured using the TG method, with the exception of the four SilLs that are a solid at room temperature. Although the diffusivities in Sills at room temperature are not as high as in silicone oil at the same $T / \eta$, a slight enhancement with the solute molecules, particularly $\mathrm{CO}$, was observed compared with that in conventional ILs and their mixtures with molecular solvents. Comparing the previous results in the PhIL system, the solute diffusivity is lower in the SilLs. However, the difference in the deviation parameter $p$ indicates that $\mathrm{CO}$ diffusion eventually becomes faster in the SilLs in low $T / \eta$ region. The similarity of the value $p$ between the silicone oils and the SilLs demonstrates that the latter inherits the characteristic solutediffusion property from the former. Measurements at various temperatures confirmed faster solute diffusion in Sills than in AlIL system even though the gap is less prominent. These findings strongly imply that the factors that control the solute diffusion differ between these types of ILs. There are three possible factors that 
can explain the faster solute diffusion, i.e., flexibility, interaction, and free volume fraction. Previous results combined with our estimation of free volume fraction imply that all the factors contribute to the difference in the diffusion.

In this study, we took a different approach to designing ILs that were applicable as reaction media and electrolytes, which involves high deviation from the SE equation, while not lowering the viscosity. While this concept was successfully realized, the viscosity properties became worse in SilLs, lowering the solute diffusivity. The slight diffusion enhancement observed for $\mathrm{CO}$ in Sills was not able to overcome the increase in viscosity; therefore, the solute diffusivity of the SilLs were lower than those of AlILs at the same temperature. This is due to the bulkiness of $\mathrm{Si}$ or $\mathrm{Si}-\mathrm{O}-\mathrm{Si}$ groups with short chain length which obscures the three above mentioned factors. Berg et al. investigated rotational diffusion of anthracene in polydimethylsiloxanes (silicone oil) with different molecular weight to estimate "nanoviscosity". 13-14 Nanovisocisty of the polymer that would be associated with torsional barriers diverges from macroviscosity in the oligomeric region. In this scenario, ILs with a longer siloxane chain are expected to show significant enhancements in solute diffusivity, which is currently under investigation in our group.

\section{Supporting Information}

Procedure of preparation of [propylSiIm] [FSI], ${ }^{1} \mathrm{H},{ }^{13} \mathrm{C}$, and ${ }^{19} \mathrm{~F}$ NMR analyses of SilLs, diffusion coefficient and $p$ value data, ion structures used for ion volume calculation, the SE plots incorporated with the GW theory. This material is available free of charge via the Internet at http://pubs.acs.org.

\section{Acknowledgements}

This work was promoted by JSPS KAKENHI Grant Number 23560918, and by COI program "Construction of next-generation infrastructure using innovative materials. Realization of a safe and secure society that can coexist with the Earth for centuries" supported by MEXT and JST.

\section{References}

(1) MacFarlane, D. R.; Golding, J.; Forsyth, S.; Forsyth, M.; Deacon, G. B. Low viscosity ionic liquids based on organic salts of the dicyanamide anion. Chem. Commun. 2001, 1430-1431.

(2) Ishikawa, M.; Sugimoto, T.; Kikuta, M.; Ishiko, E.; Kono, M. Pure ionic liquid electrolytes compatible with a graphitized carbon negative electrode in rechargeable lithium-ion batteries. J. Power Sources 2006, $162,658-662$. 
(3) Fang, S.; Yang, L.; Wei, C.; Peng, C.; Tachibana, K.; Kamijima, K. Low-viscosity and low-melting point asymmetric trialkylsulfonium based ionic liquids as potential electrolytes. Electrochem. Commun. 2007, 9, 2696-2702.

(4) Tsunashima, K.; Sugiya, M. Physical and electrochemical properties of low-viscosity phosphonium ionic liquids as potential electrolytes. Electrochem. Commun. 2007, 9, 2353-2358.

(5) Zhou, Z. B.; Matsumoto, H.; Tatsumi, K. Low-melting, low-viscous, hydrophobic ionic liquids: Aliphatic quaternary ammonium salts with perfluoroalkyltrifluoroborates. Chem. Eur. J. 2005, 11, 752-766.

(6) Stein, A. D.; Hoffmann, D. A.; Marcus, A. H.; Leezenberg, P. B.; Frank, C. W.; Fayer, M. D. Dynamics in poly(dimethylsiloxane) melts: fluorescence depolarization measurements of probe chromophore orientational relaxation. J. Phys. Chem. 1992, 96, 5255-5263.

(7) Chu, D. Y.; Thomas, J. K. Photophysical studies of molecular mobility in polymer films and bulk polymers. 2. Quenching of pyrene fluorescence by phthalic anhydride in bulk poly(dimethylsiloxanes). $J$. Phys. Chem. 1989, 93, 6250-6257.

(8) Tamai, Y.; Tanaka, H.; Nakanishi, K. Molecular Simulation of Permeation of Small Penetrants through Membranes. 1. Diffusion Coefficients. Macromolecules 1994, 27, 4498-4508.

(9) Hofmann, D.; Fritz, L.; Ulbrich, J.; Paul, D. Molecular simulation of small molecule diffusion and solution in dense amorphous polysiloxanes and polyimides. Comput. Theor. Polym. Sci. 2000, 10, 419-436.

(10) Charati, S. G.; Stern, S. A. Diffusion of Gases in Silicone Polymers: Molecular Dynamics Simulations. Macromolecules 1998, 31, 5529-5535.

(11) Cherdhirankorn, T.; Harmandaris, V.; Juhari, A.; Voudouris, P.; Fytas, G.; Kremer, K.; Koynov, K. Fluorescence Correlation Spectroscopy Study of Molecular Probe Diffusion in Polymer Melts.

Macromolecules 2009, 42, 4858-4866.

(12) Millen, W.; Hawkes, S. Diffusion of n-Alkanes in Poly(dimethylsiloxanes). Macromolecules 1977, 10, 1250-1252.

(13) Shirota, H.; Castner, E. W. Why are viscosities lower for ionic liquids with -CH2Si(CH3)3 vs $\mathrm{CH} 2 \mathrm{C}(\mathrm{CH} 3) 3$ substitutions on the imidazolium cations? J. Phys. Chem. B 2005, 109, 21576-85.

(14) Shirota, H.; Wishart, J. F.; Castner, E. W. Intermolecular interactions and dynamics of room temperature ionic liquids that have silyl- and siloxy-substituted imidazolium cations. J. Phys. Chem. B 2007, 111, 4819-29.

(15) Chavan, S. N.; Mandal, D. Combined Effect of Ether and Siloxane Substituents on Imidazolium Ionic Liquids. RSC Advances 2015.

(16) Li, P.; Wang, W.; Du, Z.; Wang, G.; Li, E.; Li, X. Adsorption and aggregation behavior of surface active trisiloxane room-temperature ionic liquids. Colloids Surf. Physicochem. Eng. Aspects 2014, 450, 5258.

(17) Tan, J.; Feng, S. Effect of Counterions on Micellization of Pyrrolidinium Based Silicone Ionic Liquids in Aqueous Solutions. J. Chem. Eng. Data 2014, 59, 1830-1834.

(18) Weng, W.; Zhang, Z.; Lu, J.; Amine, K. A disiloxane-functionalized phosphonium-based ionic liquid as electrolyte for lithium-ion batteries. Chem. Commun. 2011, 47, 11969-11971. 
(19) Du, Z.; Li, E.; Cao, Y.; Li, X.; Wang, G. Synthesis of trisiloxane-tailed surface active ionic liquids and their aggregation behavior in aqueous solution. Colloids Surf. Physicochem. Eng. Aspects 2014, 441, 744 751.

(20) Bertasi, F.; Negro, E.; Vezzù, K.; Di Noto, V. Iodide-conducting plastic crystals based on N,Ndimethyl-2-(methylsilyloxy) ethanaminium cations (MESEAn+) for application in dye-sensitized solar cells. Int. J. Hydrogen Energy 2014, 39, 2896-2903.

(21) Lee, S.; Jeon, Y.; Lim, Y.; Cho, Y.; Lee, S.; Kim, W. Novel pyridinium iodide containing siloxane high performance electrolyte for dye-sensitized solar cell. Bull. Korean Chem. Soc. 2013, 34, 2583-2588.

(22) Lee, S.; Jeon, Y.; Lim, Y.; Hossain, M. A.; Lee, S.; Cho, Y.; Ju, H.; Kim, W. A new siloxane containing imidazolium iodide as electrolyte for dye-sensitized solar cell. Electrochim. Acta 2013, 107, 675680.

(23) Lee, S. H.; Lim, Y. D.; Seo, D. W.; Hossain, M. A.; Jang, H. H.; Lee, H. C.; Kim, W. G. Novel cyclic sulfonium iodide containing siloxane high performance electrolyte for dye-sensitized solar cell. J. Ind. Eng. Chem. 2013, 19, 322-326.

(24) Blasucci, V.; Dilek, C.; Huttenhower, H.; John, E.; Llopis-Mestre, V.; Pollet, P.; Eckert, C. A.; Liotta, C. L. One-component, switchable ionic liquids derived from siloxylated amines. Chem. Commun. 2009, 116118.

(25) Lewcenko, N. A.; Byrnes, M. J.; Cheng, Y.-B.; Zakeeruddin, S. M.; Gratzel, M.; Spiccia, L. Alkylpyrrolidiniumtrialkoxysilyl iodides as organic iodide sources for dye-sensitised solar cells. Chem. Commun. 2008, 3852-3854.

(26) Bulut, S.; Ab Rani, M. A.; Welton, T.; Lickiss, P. D.; Krossing, I. Preparation of [Al(hfip)4]--Based Ionic Liquids with Siloxane-Functionalized Cations and Their Physical Properties in Comparison with Their [Tf2N]- Analogues. ChemPhysChem 2012, 13, 1802-1805.

(27) Niedermeyer, H.; Ab Rani, M. A.; Lickiss, P. D.; Hallett, J. P.; Welton, T.; White, A. J. P.; Hunt, P. A. Understanding siloxane functionalised ionic liquids. Phys. Chem. Chem. Phys. 2010, 12, 2018-2029. (28) Nishiyama, Y.; Fukuda, M.; Terazima, M.; Kimura, Y. Study of the translational diffusion of the benzophenone ketyl radical in comparison with stable molecules in room temperature ionic liquids by transient grating spectroscopy. J. Chem. Phys. 2008, 128, 164514.

(29) Kimura, Y.; Kida, Y.; Matsushita, Y.; Yasaka, Y.; Ueno, M.; Takahashi, K. Universality of Viscosity Dependence of Translational Diffusion Coefficients of Carbon Monoxide, Diphenylacetylene, and Diphenylcyclopropenone in Ionic Liquids under Various Conditions. J. Phys. Chem. B 2015, 119, 80968103.

(30) Nishiyama, Y.; Terazima, M.; Kimura, Y. Charge Effect on the Diffusion Coefficient and the Bimolecular Reaction Rate of Diiodide Anion Radical in Room Temperature Ionic Liquids. J. Phys. Chem. B 2009, 113, 5188-5193.

(31) Frisch, M. J.; Trucks, G. W.; Schlegel, H. B.; Scuseria, G. E.; Robb, M. A.; Cheeseman, J. R.; Montgomery, J. J. A.; Vreven, T.; Kudin, K. N.; Burant, J. C., et al. Gaussian 03, Revision B.04, Gaussian, Inc: Wallingford CT, 2004.

(32) Becke, A. D. Density - functional thermochemistry. III. The role of exact exchange. J. Chem. Phys. 1993, $98,5648-5652$. 
(33) Lee, C.; Yang, W.; Parr, R. G. Development of the Colle-Salvetti correlation-energy formula into a functional of the electron density. Phys. Rev. B 1988, 37, 785-789.

(34) Miehlich, B.; Savin, A.; Stoll, H.; Preuss, H. Results obtained with the correlation energy density functionals of becke and Lee, Yang and Parr. Chem. Phys. Lett. 1989, 157, 200-206.

(35) Parsons, D. F.; Ninham, B. W. Ab Initio Molar Volumes and Gaussian Radii. J. Phys. Chem. A 2009, $113,1141-1150$.

(36) Endo, T.; Murata, H.; Imanari, M.; Mizushima, N.; Seki, H.; Sen, S.; Nishikawa, K. A Comparative Study of the Rotational Dynamics of PF6- Anions in the Crystals and Liquid States of 1-Butyl-3methylimidazolium Hexafluorophosphate : Results from 31P NMR Spectroscopy. J. Phys. Chem. B 2013, 117, 326-332.

(37) Chung, S. H.; Lopato, R.; Greenbaum, S. G.; Shirota, H.; Castner, E. W.; Wishart, J. F. Nuclear Magnetic Resonance Study of the Dynamics of Imidazolium Ionic Liquids with $-\mathrm{CH} 2 \mathrm{Si}(\mathrm{CH} 3) 3$ vs -CH2C(CH3)3 Substituents广๋. J. Phys. Chem. B 2007, 111, 4885-4893.

(38) Tokuda, H.; Hayamizu, K.; Ishii, K.; Susan, M. A. B. H.; Watanabe, M. Physicochemical properties and structures of room temperature ionic liquids. 1. Variation of anionic species. J. Phys. Chem. B 2004, 108, 16593-16600.

(39) Noda, A.; Hayamizu, K.; Watanabe, M. Pulsed-Gradient Spin - Echo 1 H and 19F NMR Ionic Diffusion Coefficient, Viscosity, and Ionic Conductivity of Non-Chloroaluminate Room-Temperature Ionic Liquids. J. Phys. Chem. B 2001, 4603-4610.

(40) Tokuda, H.; Hayamizu, K.; Ishii, K.; Susan, M. A. B. H.; Watanabe, M. Physicochemical properties and structures of room temperature ionic liquids. 2. Variation of alkyl chain length in imidazolium cation. $J$. Phys. Chem. B 2005, 109, 6103-10.

(41) Tokuda, H.; Ishii, K.; Susan, M. A. B. H.; Tsuzuki, S.; Hayamizu, K.; Watanabe, M. Physicochemical properties and structures of room-temperature ionic liquids. 3. Variation of cationic structures. J. Phys. Chem. B 2006, 110, 2833-9.

(42) Seki, S.; Kobayashi, T.; Kobayashi, Y.; Takei, K.; Miyashiro, H.; Hayamizu, K.; Tsuzuki, S.; Mitsugi, T.; Umebayashi, Y. Effects of cation and anion on physical properties of room-temperature ionic liquids. $J$. Mol. Liq. 2010, 152, 9-13.

(43) Reiter, J.; Paillard, E.; Grande, L.; Winter, M.; Passerini, S. Physicochemical properties of Nmethoxyethyl-N-methylpyrrolidinum ionic liquids with perfluorinated anions. Electrochim. Acta 2013, 91, 101-107.

(44) Kowert, B. A.; Turner Ii, R. M.; Caldwell, C. V. C. Diffusion of 1-alkenes and cyclohexene in alkane solvents. Chem. Phys. 2008, 344, 114-120.

(45) Su, J. T.; Duncan, P. B.; Momaya, A.; Jutila, A.; Needham, D. The effect of hydrogen bonding on the diffusion of water in n-alkanes and n-alcohols measured with a novel single microdroplet method. J. Chem. Phys. 2010, 132, 044506.

(46) Kaintz, A.; Baker, G.; Benesi, A.; Maroncelli, M. Solute Diffusion in Ionic Liquids, NMR Measurements and Comparisons to Conventional Solvents. J. Phys. Chem. B 2013, 117, 11697-11708.

(47) Trejo González, J. A.; Longinotti, M. P.; Corti, H. R. Diffusion-Viscosity Decoupling in Supercooled Glycerol Aqueous Solutions. J. Phys. Chem. B 2015, 119, 257-262. 
(48) Köddermann, T.; Ludwig, R.; Paschek, D. On the validity of Stokes-Einstein and Stokes-EinsteinDebye relations in ionic liquids and ionic-liquid mixtures. ChemPhysChem 2008, 9, 1851-8.

(49) Alam, T. M.; Dreyer, D. R.; Bielawski, C. W.; Ruoff, R. S. Combined Measurement of Translational and Rotational Diffusion in Quaternary Acyclic Ammonium and Cyclic Pyrrolidinium Ionic Liquids. $J$. Phys. Chem. B 2013, 117, 1967-1977.

(50) Becker, S. R.; Poole, P. H.; Starr, F. W. Fractional Stokes-Einstein and Debye-Stokes-Einstein Relations in a Network-Forming Liquid. Phys. Rev. Lett. 2006, 97, 055901.

(51) Xu, L.; Mallamace, F.; Yan, Z.; Starr, F. W.; Buldyrev, S. V.; Eugene Stanley, H. Appearance of a fractional Stokes-Einstein relation in water and a structural interpretation of its onset. Nat. Phys. 2009, 5, 565569.

(52) Harris, K. R. The fractional Stokes-Einstein equation: Application to Lennard-Jones, molecular, and ionic liquidsa). J. Chem. Phys. 2009, 131, 054503.

(53) Ferguson, L.; Scovazzo, P. Solubility, diffusivity, and permeability of gases in phosphonium-based room temperature ionic liquids: Data and correlations. Ind. Eng. Chem. Res. 2007, 46, 1369-1374.

(54) Gierer, A.; Wirt, K. Molekulare Theorie der Mikroreibung. Z. Naturforsch., A: Phys. Sci. 1953, 8, 532-538.

(55) Cohen, M. H.; Turnbull, D. Molecular Transport in Liquids and Glasses. J. Chem. Phys. 1959, 31, 1164-1169.

(56) Součková, M.; Klomfar, J.; Pátek, J. Measurements and group contribution analysis of $0.1 \mathrm{MPa}$ densities for still poorly studied ionic liquids with the [PF6] and [NTf2] anions. J. Chem. Thermodyn. 2014, 77, 31-39.

(57) Gomes de Azevedo, R.; Esperança, J. M. S. S.; Szydlowski, J.; Visak, Z. P.; Pires, P. F.; Guedes, H. J. R.; Rebelo, L. P. N. Thermophysical and thermodynamic properties of ionic liquids over an extended pressure range: [bmim][NTf2] and [hmim][NTf2]. J. Chem. Thermodyn. 2005, 37, 888-899. 
Table 1 . Viscosity and density of the solvents

\begin{tabular}{|c|c|c|}
\hline IL & $\begin{array}{c}\text { Viscosity at } \\
296 \mathrm{~K} / \mathrm{mPa} \mathrm{s}\end{array}$ & $\begin{array}{c}\text { Density at } \\
298.2 \mathrm{~K} / \mathrm{g} \\
\mathrm{cm}^{-3}\end{array}$ \\
\hline [propylSiIm][FSI] & 94.5 & 1.248 \\
\hline [propylSiIm][TFSI] & 131.5 & 1.317 \\
\hline [propylSiIm][BETI] & 298.2 & 1.394 \\
\hline [propylSilm][PF6] & 2158 & 1.252 \\
\hline [SiOSiIm][FSI] & 76.5 & 1.206 \\
\hline [SiOSiIm][TFSI] & 87.1 & 1.324 \\
\hline [SiOSiIm][BETI] & 132.4 & 1.367 \\
\hline$[\mathrm{SiOSiIm}]\left[\mathrm{PF}_{6}\right]$ & 548.6 & 1.237 \\
\hline [propylSiPyrr][FSI] & - & - \\
\hline [propylSiPyrr][TFSI] & 325.9 & 1.299 \\
\hline [propylSiPyrr][BETI] & 871.5 & 1.374 \\
\hline [propylSiPyrr][PF 6$]$ & - & - \\
\hline [SiOSiPyrr][FSI] & 122.6 & 1.218 \\
\hline [SiOSiPyrr][TFSI] & 174.7 & 1.290 \\
\hline [SiOSiPyrr][BETI] & - & - \\
\hline$[\mathrm{SiOSiPyrr}]\left[\mathrm{PF}_{6}\right]$ & - & - \\
\hline$[\mathrm{P} 1,3][\mathrm{TFSI}]$ & 70.3 & 1.410 \\
\hline$[\mathrm{P} 1,4][\mathrm{TFSI}]$ & 79.7 & 1.395 \\
\hline$[\mathrm{AEIm}][\mathrm{TFSI}]$ & 30.0 & 1.456 \\
\hline KF-96-10CS & 10.3 & $0.935^{\mathrm{a}}$ \\
\hline KF-96-50CS & 50.3 & $0.960^{\mathrm{a}}$ \\
\hline KF-96-100CS & 99.8 & $0.965^{\mathrm{a}}$ \\
\hline KF-96-500CS & 496.0 & $0.970^{\mathrm{a}}$ \\
\hline
\end{tabular}

${ }^{a}$ from material specifications of the products 
Table 2. Cation and anion volumes. The ion structures used for calculations are shown in Figure S1. Free volume fractions were derived from eq. (9). Data of [BMIm][TFSI] and [EMIm] [TFSI] are also listed, the density of which were taken from the refs. ${ }^{58-59}$

\begin{tabular}{|l|c|c|c|}
\hline \multicolumn{1}{|c|}{ IL } & $\begin{array}{c}\text { Cation volume } \\
/ \AA^{3}\end{array}$ & $\begin{array}{c}\text { Anion volume } / \\
\AA^{3}\end{array}$ & $\begin{array}{c}\text { Free volume } \\
\text { fraction }\end{array}$ \\
\hline [propylSiIm][FSI] & $210.36 \pm 6.21$ & $100.40 \pm 4.77$ & $0.382 \pm 0.016$ \\
\hline [propylSiIm][TFSI] & $210.36 \pm 6.21$ & $149.32 \pm 7.00$ & $0.403 \pm 0.016$ \\
\hline [propylSiIm][BETI] & $210.36 \pm 6.21$ & $197.05 \pm 8.46$ & $0.408 \pm 0.015$ \\
\hline [propylSiIm][PF $]$ & $210.36 \pm 6.21$ & $69.93 \pm 6.01$ & $0.383 \pm 0.019$ \\
\hline [SiOSiIm][FSI] & $246.90 \pm 13.93$ & $100.40 \pm 4.77$ & $0.405 \pm 0.025$ \\
\hline [SiOSiIm][TFSI] & $246.90 \pm 13.93$ & $149.32 \pm 7.00$ & $0.397 \pm 0.024$ \\
\hline [SiOSiIm][BETI] & $246.90 \pm 13.93$ & $197.05 \pm 8.46$ & $0.414 \pm 0.022$ \\
\hline [SiOSiIm][PF 6$]$ & $246.90 \pm 13.93$ & $69.93 \pm 6.01$ & $0.393 \pm 0.019$ \\
\hline [propylSiPyr][FSI] & $219.98 \pm 9.19$ & $100.40 \pm 4.77$ & - \\
\hline [propylSiPyr][TFSI] & $219.98 \pm 9.19$ & $149.32 \pm 7.00$ & $0.399 \pm 0.019$ \\
\hline [propylSiPyr][BETI] & $219.98 \pm 9.19$ & $197.05 \pm 8.46$ & $0.406 \pm 0.018$ \\
\hline [propylSiPyr][PF $]$ & $219.98 \pm 9.19$ & $69.93 \pm 6.01$ & - \\
\hline [SiOSiPyr][FSI] & $258.07 \pm 11.22$ & $100.40 \pm 4.77$ & $0.384 \pm 0.021$ \\
\hline [SiOSiPyr][TFSI] & $258.07 \pm 11.22$ & $149.32 \pm 7.00$ & $0.399 \pm 0.020$ \\
\hline [SiOSiPyr][BETI] & $258.07 \pm 11.22$ & $197.05 \pm 8.46$ & - \\
\hline [SiOSiPyr][PF $]$ & $258.07 \pm 11.22$ & $69.93 \pm 6.01$ & - \\
\hline [P1,3][TFSI] & $143.78 \pm 5.20$ & $149.32 \pm 7.00$ & $0.391 \pm 0.018$ \\
\hline [P1,4][TFSI] & $158.83 \pm 9.81$ & $149.32 \pm 7.00$ & $0.387 \pm 0.024$ \\
\hline [BMIm][TFSI] & $144.31 \pm 4.76$ & $149.32 \pm 7.00$ & $0.394 \pm 0.017$ \\
\hline [EMIm][TFSI] & $112.67 \pm 6.30$ & $149.32 \pm 7.00$ & $0.387 \pm 0.022$ \\
\hline [AEIm][TFSI] & $141.63 \pm 9.66$ & $149.32 \pm 7.00$ & $0.389 \pm 0.025$ \\
\hline
\end{tabular}


Scheme 1. Photodissociation reaction scheme of DPCP

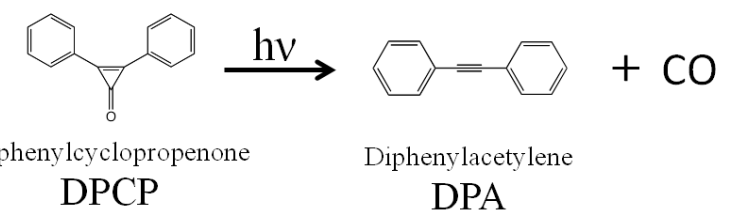




\section{FIGURE CAPTIONS}

Figure 1. Molecular structure of the cations and anions for Sills.

Figure 2. TG signals from (a) DPCP in [propylSiIm][FSI] (black and red curves represent experimental and theoretically fitted data, respectively). Utilizing eq. (2) reveals each component buried in the signal shown in (a). Diffusion coefficients of (b) CO, (c) DPA, and DPCP are estimated as the slope of $1 / \tau$ versus $q^{2}$.

Figure 3. Diffusion coefficients of (a) CO, (b) DPA, and (c) DPCP against $T / \eta$. Closed and open symbols are data obtained here and from the refs, ${ }^{31-32}$ respectively (blue: silicone oils, red: SiILs, black: AlILs, molecular solvents and their mixtures, and green: $\mathrm{PhILs}$ and their mixtures with molecular solvents). Two outliers in (a) are the data in ethylene glycol and glycerol. Note that the data in the references include diffusion in AlILs at high pressure. Fitted curves for each plot are from eq. (6). Grey lines represent the diffusion coefficients predicted from the Stokes-Einstein equation, assuming $C=6$ (solid) and $C=4$ (dashed). The solute hydrodynamic radii used were $1.86 \AA$ for $\mathrm{CO}, 3.50 \AA$ for DPA, and $3.54 \AA$ for DPCP, taken from the literature. ${ }^{31}$ These values are also applied in Figures 4, S2, and S3.

Figure 4. Temperature-dependent diffusion coefficients of (a) CO, (b) DPA, and (c) DPCP against $T / \eta$ in silicone oils (closed blue), two SiILs ([propylSiIm][TFSI] and [SiOSiIm][TFSI], closed red), three AlILs ([BMIm][TFSI], [EMIm][TFSI], and [AEIm][TFSI], closed black, the former two are from the ref ${ }^{31}$ ), and PhILs (from the ref, ${ }^{31}$ closed green). Open small symbols are the data at room temperature taken from Figure 3. Fitted curves for each plot (solid for various temperature and dashed for room temperature) are from eq. (6). Grey lines represent the diffusion coefficients predicted from the Stokes-Einstein equation, assuming $C$ $=6$ (solid) and $C=4$ (dashed). 
Figure 1. Molecular structures of the cations and anions of SilLs.

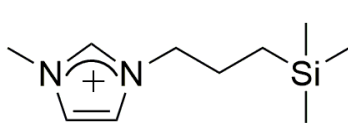

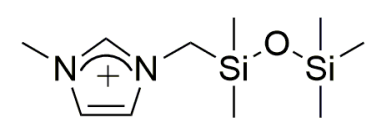<smiles>C[N+]1(CCC[Si](C)(C)C)CCCC1</smiles>

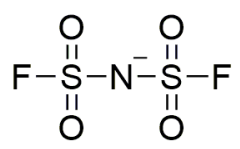

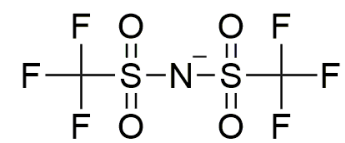

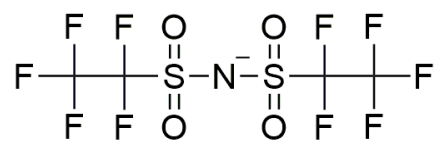

$\mathrm{PF}_{6}^{-}$ 
Figure 2. TG signals from (a) DPCP in [propylSiIm][FSI] (black and red curves represent experimental and theoretically fitted data, respectively). Utilizing eq. (2) reveals each component buried in the signal shown in (a). Diffusion coefficients of (b) CO, (c) DPA, and DPCP are estimated as the slope of $1 / \tau$ versus $q^{2}$.

(a)

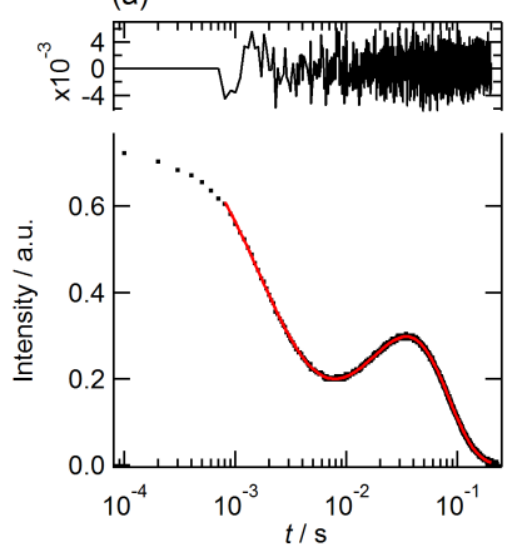

(b)

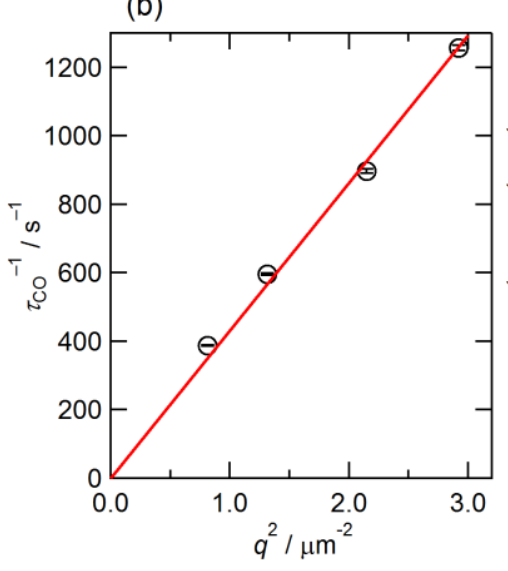

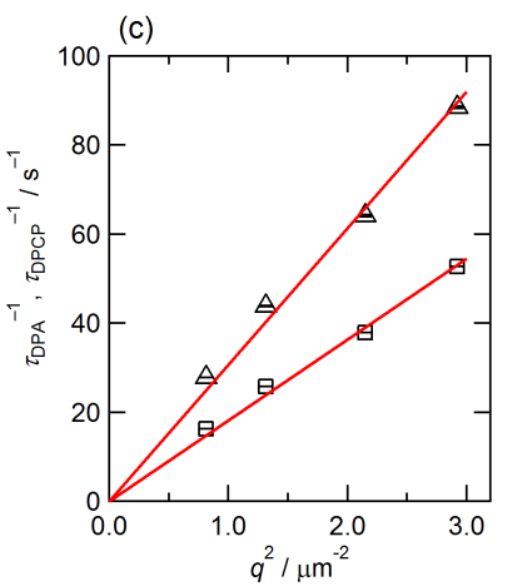


Figure 3. Diffusion coefficients of (a) CO, (b) DPA, and (c) DPCP against $T / \eta$. Closed and open symbols are data obtained here and from the refs, ${ }^{31-32}$ respectively (blue: silicone oils, red: Sills, black: AllLs, molecular solvents and their mixtures, and green: $\mathrm{PhILs}$ and their mixtures with molecular solvents). Two outliers in (a) are the data in ethylene glycol and glycerol. Note that the data in the references include diffusion in AlILs at high pressure. Fitted curves for each plot are from eq. (6). Grey lines represent the diffusion coefficients predicted from the Stokes-Einstein equation, assuming $C=6$ (solid) and $C=4$ (dashed). The solute hydrodynamic radii used were $1.86 \AA$ for $\mathrm{CO}, 3.50 \AA$ for DPA, and $3.54 \AA$ for DPCP, taken from the literature. ${ }^{31}$ These values are also applied in Figures 4, S2, and S3.

(a)

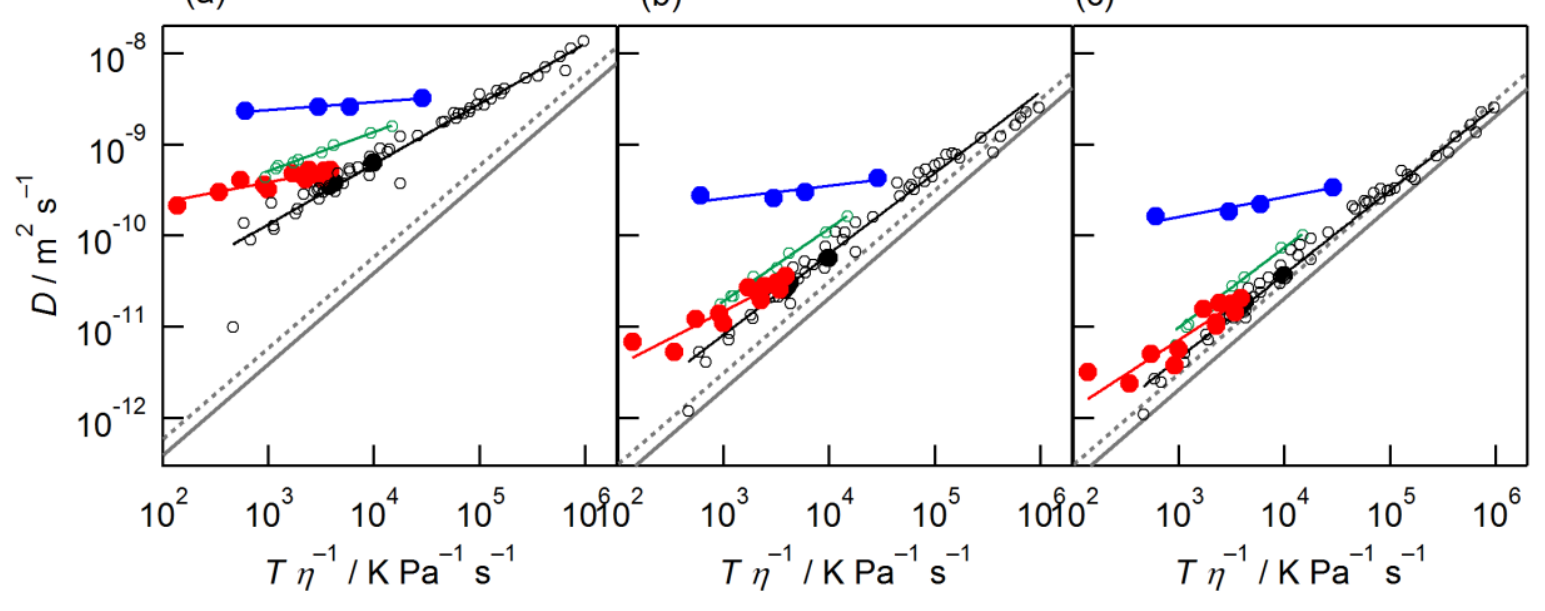


Figure 4. Temperature-dependent diffusion coefficients of (a) CO, (b) DPA, and (c) DPCP against $T / \eta$ in silicone oils (closed blue), two SiILs ([propylSiIm][TFSI] and [SiOSiIm][TFSI], closed red), three AlILs ([BMIm][TFSI], [EMIm][TFSI], and [AEIm][TFSI], closed black, the former two are from the ref ${ }^{31}$ ), and PhILs (from the ref, ${ }^{31}$ closed green). Open small symbols are the data at room temperature taken from Figure 3. Fitted curves for each plot (solid for various temperature and dashed for room temperature) are from eq. (6). Grey lines represent the diffusion coefficients predicted from the Stokes-Einstein equation, assuming $C$ $=6$ (solid) and $C=4$ (dashed).

(a)

(b)

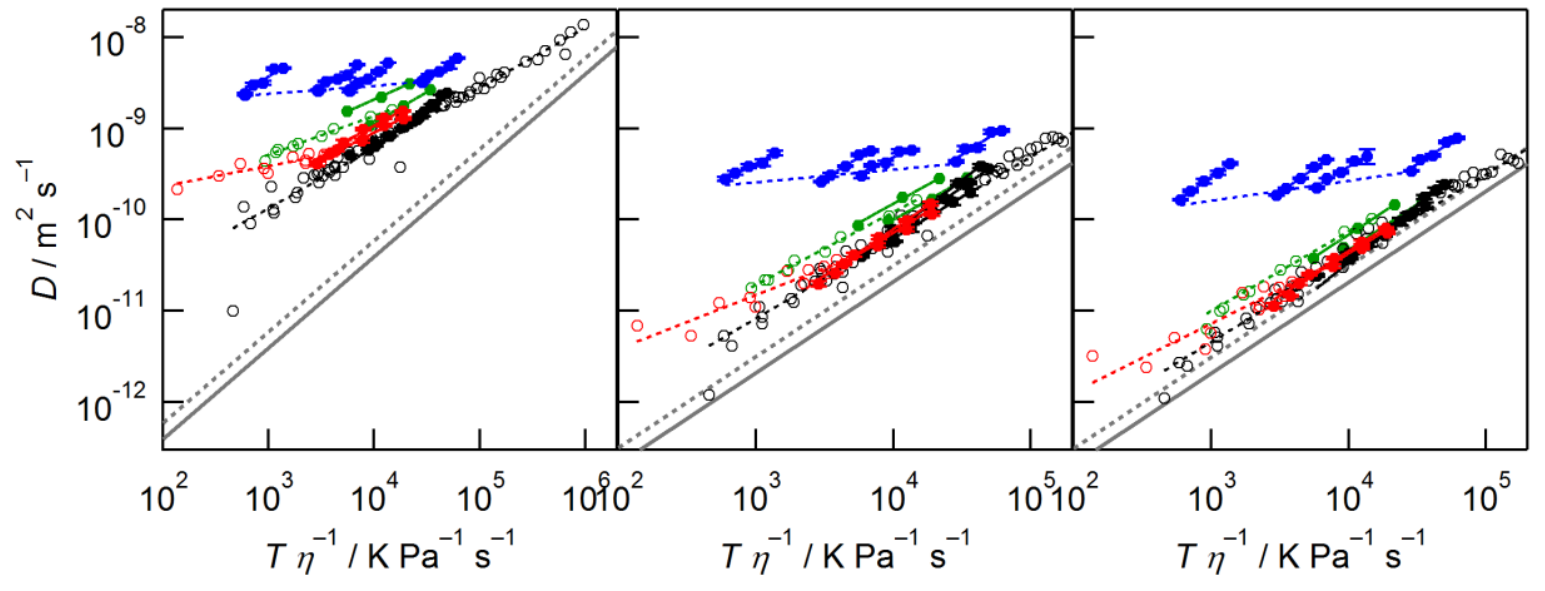


TOC Graphic

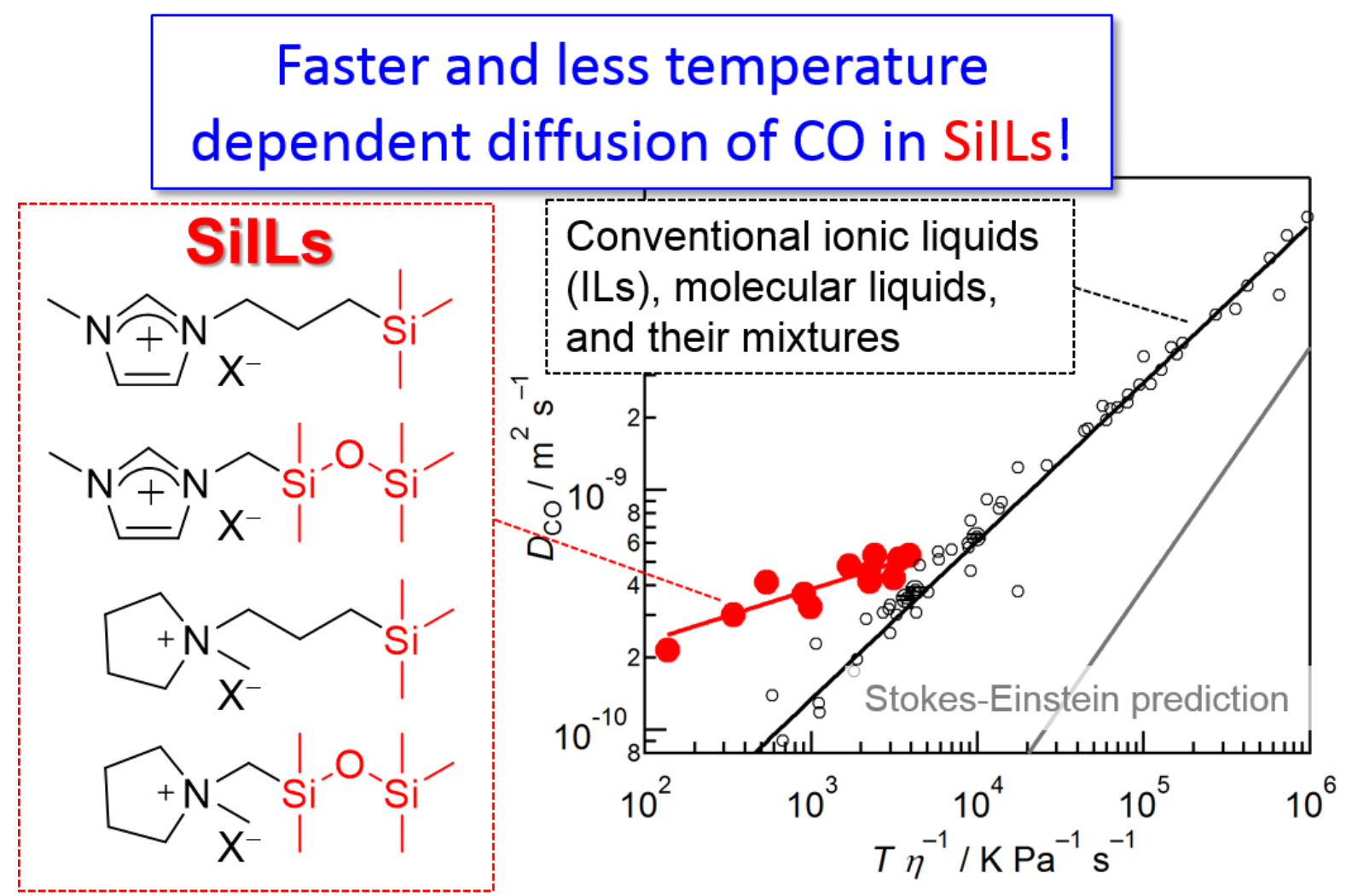

